\title{
Rapid microRNA detection using power-free microfluidic chip: coaxial stacking effect enhances the sandwich hybridization $\dagger$
}

\author{
Hideyuki Arata, $\ddagger$ Hiroshi Komatsu, Aishan Han, Kazuo Hosokawa* and Mizuo Maeda \\ Received 24th November 2011, Accepted 6th May 2012 \\ DOI: 10.1039/c2an16154k
}

\begin{abstract}
We present a new method for rapid microRNA detection with a small volume of sample using the power-free microfluidic device driven by degassed PDMS. Target microRNA was detected by sandwich hybridization taking advantage of the coaxial stacking effect. This method allows us to detect miR-21 in 20 min with a $0.5 \mu \mathrm{L}$ sample volume at a limit of detection of $0.62 \mathrm{nM}$. Since microRNAs can act as cancer markers, this method might substantially contribute to future point-of-care cancer diagnosis.
\end{abstract}

MicroRNAs (miRNAs) are small non-protein-coding singlestranded RNAs of typically 18 to 25 bases. ${ }^{1}$ Since miRNAs expression profiling can detect or even classify cancer in human body, detection of miRNA is one of the key topics in the new generation of cancer research. ${ }^{1-6}$ Some of miRNAs circulate in human body fluid with extremely low concentration at the early stage of cancer, therefore, highly sensitive miRNA detection with portable device will open a new field of point-of-care (POC) cancer diagnosis. ${ }^{46}$ There are several requirements in POC cancer diagnosis. Several kinds of sequences should be detected at once, because each cancer has a few number of corresponding marker miRNAs. Detection time should be less than an hour with a sample volume of less than a few microliters. The device should be portable and simple to operate without the need for bulky equipment, for use in resource-poor environments. Various techniques, such as quantitative real-time PCR (qPCR), sequencing and microarrays, ${ }^{7}$ allow profiling miRNAs and several successful studies to detect miRNA with high sensitivity have been reported..$^{-11}$ qPCR has an advantage of ultra-sensitive quantification besides microarray allows high-throughput screening. However, detection time, required sample volume, simplicity and portability of the device have not yet reached the requirements for POC diagnosis. To meet those requirements, the use of microfluidic devices, which conveys sample RNA molecules to immobilized probe DNA by microchannels, can be an attractive choice because it can reduce the assay time. This effect is attributed to a shortening of the distance that the sample molecules must travel to the solid-phase by diffusion. ${ }^{12}$ One of the major challenges to be addressed for this microfluidic approach

Bioengineering Laboratory, RIKEN, 2-1 Hirosawa, Wako, Saitama 351-0198, Japan. E-mail: k-hoso@riken.go.jp; Tel: +81-48-467-9312

$\dagger$ Electronic supplementary information (ESI) available. See DOI: 10.1039/c2an16154k

† Present address: JST-ERATO Higashiyama Live-Holonics Project and Graduate School of Science, Nagoya University, Japan. has been the detection of short and non-labelled miRNA by surface sandwich hybridization. In this report, we demonstrate that the coaxial stacking effect ${ }^{13-16}$ can be effectively used for achieving this type of detection. As a detection platform, we adopted our power-free PDMS microfluidic chip, ${ }^{17,18}$ which eliminates the need for external power sources for fluid pumping, as a straightforward strategy of rapid miRNA detection towards future POC cancer diagnosis.

Power-free poly(dimethylsiloxane) (PDMS) microfluidic device was fabricated as reported elsewhere ${ }^{17,18}$ with an additional process of selective immobilization of probe DNA on glass plates. This microfluidic device allows self-pumping by PDMS air absorption without connecting to an exterior power supply. Two PDMS microchannels were used: first for the selective patterning of probe DNA and second for miRNA detection. Symbols and sequences of oligonucleotides, probe DNAs and miRNAs, used in this study are listed in Table 1. The device fabrication process is as follows. First, glutaraldehyde (Wako) was incubated on aminated glass plates (SD00011, $25.4 \mathrm{~mm}$ $\times 76.2 \mathrm{~mm}$, Matsunami Glass Ind., LTD) at $37^{\circ} \mathrm{C}$ for two hours. Next, a PDMS microchannel of $100 \mu \mathrm{m}$ in width and $25 \mu \mathrm{m}$ in height was formed on the glass plate and degassed in a vacuum chamber for 40 minutes. Amino-labelled DNA (Pro21, Pro141) (Oligonucleotide, Europhins operon) was injected into the channel by power-free (a)

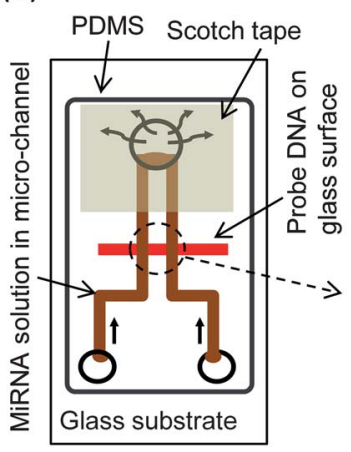

(b)

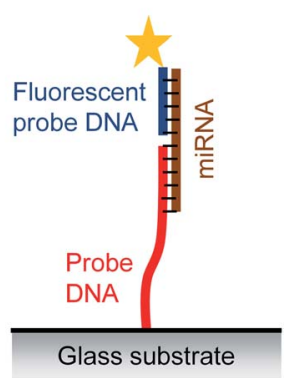

Fig. 1 (a) Schematics of the power-free microfluidic device. PDMS absorbs air in the outlet chamber thus being a self-stand pumping device. Probe DNA is immobilized onto the glass surface and microchannels convey the sample to the probe. (b) Schematics of sandwich hybridization. Half of the miRNA sequence is complementary to the fluorescent labeled probe DNA and the other half is complementary to the probe DNA immobilized on the glass surface. 
Table 1 Sequences of the oligonucleotides used in this work: DNAs (Operon Inc.) and miRNAs (Greiner Inc.)

\begin{tabular}{ll}
\hline Symbol & Sequence (from $5^{\prime}$ to $3^{\prime}$ ) \\
\hline Pro21 & $\mathrm{NH}_{2}$-TTT TTT TTT TTT TTT TCA ACA TCA GT \\
Pro141 & $\mathrm{NH}_{2}$-TTT TTT TTT TTT TTT CCA TCT TTA CC \\
ProFlu21 & CTG ATA AGC TA-Fluorescein \\
ProFlu141 & AGA CAG TGT TA-Fluorescein \\
Pro21full & $\mathrm{NH}_{2}$-TTT TTT TTT TTT TTT TCA ACA TCA GTC TGA TAA GCT A \\
MiR-21 & UAG CUU AUC AGA CUG AUG UUG A \\
MiR-141 & UAA CAC UGU CUG GUA AAG AUG G \\
MiR-ran & UGG UGC GGA GAG GGC CCA CAG U \\
MiR-21Flu & Fluorescein-UAG CUU AUC AGA CUG AUG UUG A \\
MiR-21Gap & UAG CUU AUC AGU ACU GAU GUU GA
\end{tabular}

pumping and incubated at $37^{\circ} \mathrm{C}$ for $30 \mathrm{~min}$. Finally, the PDMS microchannel was detached from the glass plate in stopping buffer ( $25 \mathrm{mM}$ Tris, $0.05 \%$ Tween 20). The glass plate was washed by ultrasonication, with both the stopping buffer and deionized water, to remove the remaining DNA on the surface. The second PDMS microchannel was fabricated and mounted onto the glass plate to form microfluidic devices with several microchannels of $100 \mu \mathrm{m}$ in width and $25 \mu \mathrm{m}$ in height (Fig. 1). MiR-21 was adopted as a model sequence since it is one of the most well-known miRNAs as cancer markers. ${ }^{19,20}$

Two protocols, "Protocol 1" and "Protocol 2", were conducted to detect miRNA by sandwich hybridization. In protocol 1 , fluorescent labelled probe DNA (ProFlu21) was pre-hybridized to miR-21 in an off-chip tube before injecting into the microchannel. In protocol 2, miR-21 was hybridized to Pro21 immobilized in the microchannel, and then ProFlu21 was injected to the microchannel to hybridize to the captured miR-21. All the hybridization processes were carried out at room temperature $\left(\sim 25^{\circ} \mathrm{C}\right)$ in a blocking solution (BS) $(0.01 \%$ Roche Blocking Reagent, $0.02 \%$ (w/v) SDS, $5 \times$ SSC, $0.05 \%$ Tween 20). Protocol 1 was carried out as follows. First, miR-21 was hybridized with ProFlu 21 of $1 \mu \mathrm{M}$ in a tube with the BS for $5 \mathrm{~min}$. During this period, $0.5 \mu \mathrm{L}$ of the fresh BS was injected into the microchannel and incubated for $3 \mathrm{~min}$. Subsequently, $0.5 \mu \mathrm{L}$ of the mixture of miR-21 and ProFlu21 sustained in the BS was injected into the channel and incubated for $5 \mathrm{~min}$. Finally, non-hybridized RNA and ProFlu21 were washed out by $1-3 \mu \mathrm{L}$ of the BS for $10 \mathrm{~min}$. Protocol 2 was carried out as follows. First, $0.5 \mu \mathrm{L}$ of BS was injected into the channel and incubated for $3 \mathrm{~min}$. Next, $0.5 \mu \mathrm{L}$ of miR-21 diluted in the BS was injected into the channel and incubated for 5 min. Subsequently, $0.5 \mu \mathrm{L}$ of $1 \mu \mathrm{M}$ ProFlu 21 in the BS was injected into the channel and incubated for $5 \mathrm{~min}$. Finally, the channel was washed out by $1-3 \mu \mathrm{L}$ of $\mathrm{BS}$ for $10 \mathrm{~min}$. Detection of multiple sequences from a single sample solution was also demonstrated by protocol 1. Specifically, the microfluidic devices with probe DNAs of two different sequences, Pro21 and Pro141, were prepared with the same protocol. The sample solutions with four combinations were prepared: miR-ran in the BS: miR-21 and miR-ran in the BS: miR141 and miR-ran in the BS: miR-21, miR-141 and miR-ran in the BS. In this demonstration, the concentration of miR-21 and miR-141 was $100 \mathrm{nM}$ and that of miR-ran was $1 \mu \mathrm{M}$. In all the experiments, channels were observed by the fluorescent microscopy and images were captured by CCD camera (Photometrix Cool SNAP HQ2). Fluorescence intensities were analysed by software (ImageJ). Three to seven data were used to plot each averages and standard deviations.
With both the protocols, results with the Pro21 patterned devices showed fluorescence intensities in the channel with miR-21 but not in the channel without miRNA (Fig. 2(a)). The fluorescence intensities decreased at lower miR-21 concentrations and showed almost no fluorescence intensity less than $0.1 \mathrm{nM}$ (Fig. 2(b)). The data points were fitted with the four-parameter logistic function ${ }^{21}$ and LOD was evaluated by the $3 \sigma$ criterion. As a result, we obtained an LOD of $0.62 \mathrm{nM}$ by protocol 1 . This is comparable with typical LOD of DNA by existing microchips for solid-phase hybridization assays. ${ }^{22-24}$ On the other hand, LOD of $5.3 \mathrm{nM}$ was obtained by protocol 2 . (a)

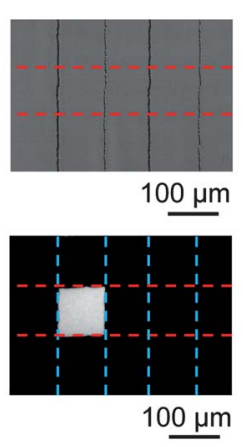

(c)

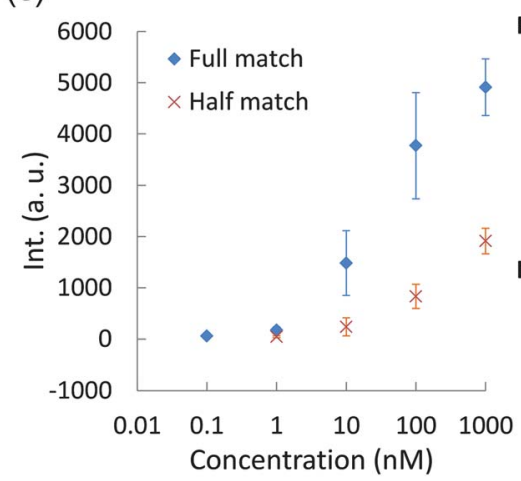

(b)

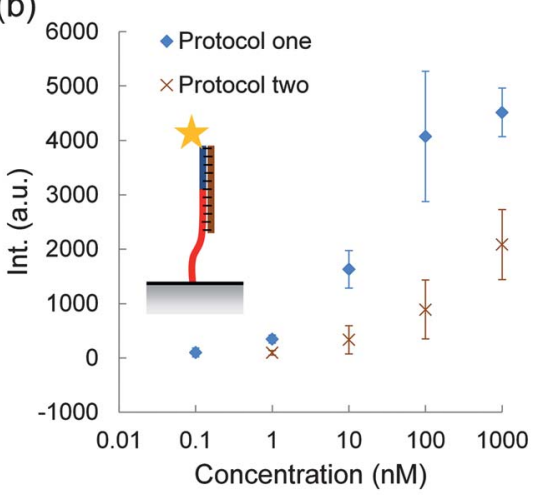

Fig. 2 (a) Bright field microscopic view (top) and fluorescent microscopic view (bottom) of microchannels by protocol 1. Red dotted lines indicate the area where probe DNA is immobilized on the glass surface. Blue dotted lines indicate the microchannels. Target miRNA was injected into the left channel and random miRNA was injected into the right channel. (b) Fluorescence intensity $v s$. miRNA concentration by the two protocols, protocol 1 and protocol 2. (c) Fluorescence intensity $v s$. fluorescent labeled miRNA concentration by probe DNA with different length. 
The lower LOD by protocol 1 can be explained by the effect of coaxial stacking-aided hybridization ${ }^{13-16}$ which is known to support oligonucleotides hybridization. It means, if we assume that this is effective also in miRNA-DNA hybridization, the affinity of ProFlu21-miR-21 hybrids to Pro21 should be higher than that of miR21 alone to Pro21. The hybridization efficiency by protocol 1 was almost the same as that of non-sandwich hybridization between fullmatched probe DNA (Pro21full) and fluorescent labelled miR-21 (MiR-21Flu) under the same condition (Fig. 2(c) Full match). This indicates that the effect of coaxial stacking allows sandwich hybridization efficiency approximately the same as non-sandwich hybridization even in short RNAs. Hybridization efficiency between halfmatched probe DNA (Pro21) and MiR-21Flu showed hybridization efficiency as low as sandwich hybridization by protocol 2 (Fig. 2(c) Half match). This suggests that the efficiency of hybridization between Pro21 and MiR-21 is the limiting factor in protocol 2.

The coaxial stacking effect can also be supported by the melting temperature $\left(T_{\mathrm{m}}\right)$ calculation. The $T_{\mathrm{m}}$ values were calculated using on-site software HyTher (see ESI $\dagger$ ). The $T_{\mathrm{m}}$ values between miR-21 and individual probes, ProFlu21 and Pro21, were $37.6{ }^{\circ} \mathrm{C}$ and $42.0^{\circ} \mathrm{C}$, respectively. On the other hand, with both of the probes, the sandwich construct including a coaxial stacking site raised the $T_{\mathrm{m}}$ value to $73.4{ }^{\circ} \mathrm{C}$ which was as high as that of intact RNA-DNA duplex with $22 \mathrm{bps}$. Those theoretical results are consistent with the experimental results that the fluorescent signals of the sandwich structure without any gap (Fig. 2(b) Protocol 1) and RNA-DNA hybrids of 22 bps (Fig. 3(c) Full match) were almost at the same level.

To further confirm this effect, both protocol 1 and protocol 2 were carried out by an artificial variant of miR-21 with additional $U$ base at the centre of the sequence (MiR-21Gap), which induces a single base gap between the two probe DNAs, while retaining the affinity to the individual probes (Fig. S1 $\dagger$ ). The results show, in both protocols, approximately the same affinity when non-labelled miR-21 was detected by protocol 2 (Fig. S2 $\dagger$ ). This can be attributed to the gap between the two probe DNAs, which eliminate the coaxial stacking effect. These results clearly indicate that the coaxial stacking effect was the major cause of the higher sensitivity with protocol 1 than that with protocol 2 . Therefore, protocol 1 with probe DNA construction without any gap between two probe DNAs is the ideal design of miRNA detection by sandwich hybridization.

The experiments of multiple sequence detection from a single sample solution resulted in fluorescence intensities only on the probe DNA of corresponding sequences in all four cases (Fig. 3). (a)

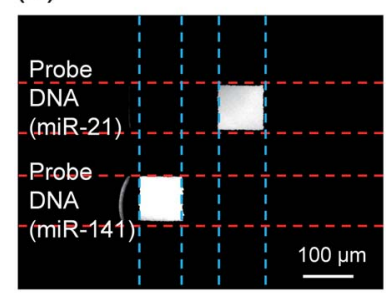

MiR-141 MiR-21 (b)

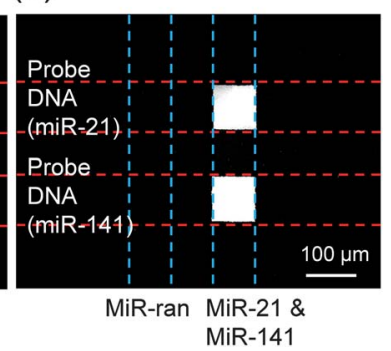

Fig. 3 Multiple sequence detection from a single sample solution. The blue lines indicate the microchannels and the red lines indicate the probe DNA patterned area. Fluorescence intensities appeared only on the corresponding probe DNAs in all four cases.
This capability of multiple sequence detection strongly supports the fact that miRNA detection by this device is a promising technology in cancer diagnosis because each cancer has only a few corresponding marker miRNA which may exclude the need of screening hundreds of sequences by conventional array chip.

\section{Conclusions}

Unlabelled miRNA was successfully detected in 20 minutes on the power-free microfluidic device. Compared to conventional methods, such as qPCR, sequencing and microarray, which usually require a few hours to an overnight incubation, detection time was drastically shortened. The LOD was as low as $0.62 \mathrm{nM}$, taking advantage of coaxial stacking-aided hybridization. Required sample volume is $0.5 \mu \mathrm{L}$ which corresponds to $300 \mathrm{amol}$ at the miRNA concentration around LOD. For practical application, there remain two technical challenges. First, LOD must further be improved. Improvement of LOD by signal amplification technique, such as laminar flow-assisted dendritic amplification (LFDA), ${ }^{25}$ or by using highly sensitive optical system might realize enough low LOD without spoiling the merits of rapidity and less amount of sample required. Second, the optical and electrical equipments for signal readout should be miniaturized. A smart way to achieve this may be the use of inexpensive consumer products like cell phones. ${ }^{26}$ Rapidness, simple operation, small required sample volume and portability of the microfluidic device are ideal advantages for point-of-care cancer diagnosis, therefore, further study might contribute to improve the healthcare environment even in resource poor environments, such as in developing countries, and may provide a positive impact in global health.

\section{Acknowledgements}

This work was partly supported by KAKENHI (22500448) and the Funding Program for World-Leading Innovative R\&D on Science and Technology.

\section{Notes and references}

1 S. S. Jeffrey, Nat. Biotechnol., 2008, 26, 400.

2 J. Lu, G. Getz, E. A. Miska, E. Alvarez-Saavedra, J. Lamb, D. Peck, A. Sweet-Cordero, B. L. Ebert, R. H. Mak, A. A. Ferrando, J. R. Dowing, T. Jacks, H. R. Horvitz and T. R. Golub, Nature, 2005, 435, 834.

3 G. A. Calin and C. M. Croce, Nat. Rev. Cancer, 2006, 6, 857.

4 P. S. Mitchell, R. K. Parkin, E. M. Kroh, B. R. Fritz, S. K. Wyman, E. L. Pogosova-Agadjanyan, A. Peterson, J. Noteboom, K. C. O'Briant, A. Allen, D. W. Lin, N. Urban, C. W. Drescher, B. S. Knudsen, D. L. Stirewalt, R. Gentleman, R. L. Vessella, P. S. Nelson, D. B. Martin and M. Tewari, Proc. Natl. Acad. Sci. U. S. A., 2008, 105, 10513 .

5 N. Kosaka, H. Iguchi and T. Ochiya, Cancer Sci., 2010, 101, 2087.

6 W. Gao, L. Liu, X. Lu and Y. Shu, Clin. Lung Cancer, 2011, 12, 14.

7 M. Baker, Nat. Methods, 2010, 7, 687.

8 P. T. Nelson, D. A. Baldwin, L. M. Scearce, J. C. Oberholtzer, J. W. Tobias and Z. Mourelatos, Nat. Methods, 2004, 1, 1.

9 E. A. Miska, E. Alvarez-Saavedra, M. Townsend, A. Yoshii, N. Sestan, P. Rakic, M. Constantine-Paton and H. R. Horvitz, Genome Biol., 2004, 5, R68.

10 M. Castoldi, S. Schmidt, V. Benes, M. W. Hentze and M. U. Muckenthaler, Nat. Protoc., 2008, 3, 321.

11 W.-J. Zhou, Y. Chen and R. M. Corn, Anal. Chem., 2011, 83, 3897.

12 D. Erickson and D. Q. Li, Anal. Chim. Acta, 2004, 507, 11

13 M. Petersheimf and D. H. Turner, Biochemistry, 1983, 22, 256.

14 D. O'Meara, P. Nilsson, P.-A. Nygren, M. Uhlen and J. Lundeberg, Anal. Biochem., 1998, 255, 195. 
15 B.-F. Yuan, X.-Y. Zhuang, Y.-H. Hao and Z. Tan, Chem. Commun., 2008,6600

16 S. Cai, C. Lau and J. Lu, Anal. Chem., 2010, 82, 7178.

17 K. Hosokawa, K. Sato, N. Ichikawa and M. Maeda, Lab Chip, 2004, 4, 181.

18 K. Hosokawa, T. Sato, Y. Sato and M. Maeda, Anal. Sci., 2010, 26, 1053.

19 M. Seike, A. Goto, T. Okano, E. D. Bowman, A. J. Schetter, I. Horikawa, E. A. Mathe, J. Jen, P. Yang, H. Sugimura, A. Gemma, S. Kudoh, C. M. Croce and C. C. Harris, Proc. Natl. Acad. Sci. U. S. A., 2009, 106, 12085.

20 V. Jazbutyte and T. Thum, Curr. Drug Targets, 2010, 11, 926.
21 P. Diamandis and T. K. Christopoulos, Immunoassay, Academic Press, San Diego, 1996.

22 K. Hatakeyama, T. Tanaka, M. Sawaguchi, A. Iwadate, Y. Mizutani, K. Sasaki, N. Tateishi and T. Matsunaga, Lab Chip, 2009, 9, 1052.

23 D. Erickson, X. Z. Liu, R. Venditti, D. Q. Li and U. J. Krull, Anal. Chem., 2005, 77, 4000.

24 H. Chen, L. Wang and P. C. H. Li, Lab Chip, 2008, 8, 826.

25 K. Hosokawa, M. Omata and M. Maeda, Anal. Chem., 2007, 79, 6000 .

26 H. Zhu, S. Mavandadi, A. F. Coskun, O. Yaglidere and A. Ozcan, Anal. Chem., 2011, 83, 6641. 